\title{
On the weak convergence for solving semistrictly quasi-monotone variational inequality problems
}

\author{
S.S. Chang ${ }^{1 *}$, Salahuddin², L. Wang ${ }^{3}$ and M. Liu ${ }^{4}$
}

"Correspondence:

changss2013@163.com

1 Center for General Education,

China Medical University, Taichung, Taiwan

Full list of author information is

available at the end of the article

\section{Springer}

\begin{abstract}
In this paper, we study the approximation problem of solutions for the semistrictly quasi-monotone variational inequalities in infinite-dimensional Hilbert spaces. We prove that the iterative sequence generated by the algorithm for solving the semistrictly quasi-monotone variational inequalities converges weakly to a solution.

MSC: 49J40; 47H09; 47J20; 54H25

Keywords: Variational inequality problems; Extragradient method; Semistrict quasi-monotonicity; Weak convergence
\end{abstract}

\section{Introduction}

The theory of variational inequalities serves as a powerful mathematical tool, which unifies important concepts in applied mathematics like systems of nonlinear equations, optimality conditions for optimization problems, complementarity problems, obstacle problems, and network equilibrium problems. Therefore, this theory has numerous applications in the fields of engineering, mathematical programming, network economics, transportation research, game theory, and regional sciences [1, 2, 13, 14, 16]. Several techniques for solving a variational inequality in infinite-dimensional spaces (such as projection method, extragradient method, Tikhonov regularization method and proximal point method) have been suggested; see, e.g., [7, 9]. The well-known gradient projection method can be successfully applied for solving strongly monotone variational inequalities and inverse strongly monotone variational inequalities $[5,7,12,17,19]$. The Tikhonov regularization and proximal point methods can serve as an efficient solution method for solving monotone variational inequalities. Korpelevich introduced the extragradient method [15], and this method was applied for solving monotone variational inequalities in infinitedimensional spaces. It is a known fact [7] that the extragradient method can be successfully applied for solving monotone variational inequalities. Recently, the extragradient method has been considered for solving variational inequalities in infinite-dimensional Hilbert spaces $[3,4,10,18]$. It is proved that if the variational inequality has solutions and the assigned mapping is monotone and Lipschitz continuous, then the iterative sequence generated by the extragradient method converges weakly to a solution.

(c) The Author(s) 2019. This article is distributed under the terms of the Creative Commons Attribution 4.0 International License (http://creativecommons.org/licenses/by/4.0/), which permits unrestricted use, distribution, and reproduction in any medium, provided you give appropriate credit to the original author(s) and the source, provide a link to the Creative Commons license, and indicate if changes were made. 
The aim of this paper is to study the approximation problem of solutions for the semistrictly quasi-monotone variational inequalities in infinite-dimensional Hilbert spaces. We prove that the iterative sequence generated by the extragradient method for solving semistrictly quasi-monotone variational inequalities converges weakly to a solution.

\section{Preliminaries}

Throughout this article we assume that $\mathcal{H}$ is a real Hilbert space with inner product $\langle\cdot, \cdot\rangle$ and induced norm $\|\cdot\|$ and $\mathcal{C}$ is a nonempty, closed and convex subset of $\mathcal{H}$.

For each $u \in \mathcal{H}$, there exists a unique point in $\mathcal{C}$, denoted by $P_{\mathcal{C}}(u)$, such that

$$
\left\|u-P_{\mathcal{C}}(u)\right\| \leq\|u-v\|, \quad \forall v \in \mathcal{C} .
$$

The operator $P_{C}$ is called the projection operator from $\mathcal{H}$ onto $\mathcal{C}$. It is well known [13] that the projection operator can be characterized by

$$
\left\langle u-P_{\mathcal{C}}(u), v-P_{\mathcal{C}}(u)\right\rangle \leq 0, \quad \forall v \in \mathcal{C} .
$$

If $P_{\mathcal{C}}$ is a projection operator of $\mathcal{H}$ onto $\mathcal{C}$, then

$$
\left\langle u-v, P_{\mathcal{C}}(u)-P_{\mathcal{C}}(v)\right| \geq\left\|P_{\mathcal{C}}(u)-P_{\mathcal{C}}(v)\right\|^{2}, \quad \forall u, v \in \mathcal{H} .
$$

Let $Q: \mathcal{C} \longrightarrow \mathcal{H}$ be a mapping. The variational inequality $V I(\mathcal{C}, Q)$ defined by $\mathcal{C}$ and $Q$ is to find a point $u^{*} \in \mathcal{C}$ such that

$$
\left\langle Q\left(u^{*}\right), u-u^{*}\right\rangle \geq 0, \quad \forall u \in \mathcal{C} .
$$

The solution set of $(2.2)$ is denoted by $\operatorname{Sol}(\mathcal{C}, Q)$.

It is known (see, for instance, [6]) that the previous problem is closely related to finding a point $u^{*} \in \mathcal{C}$ such that

$$
\left\langle Q(u), u-u^{*}\right\rangle \geq 0, \quad \forall u \in \mathcal{C} .
$$

Following [6], we shall call problem (2.3) the dual variational inequality problem $(\operatorname{DVI}(\mathcal{C}$, Q)) of (2.2).

Definition 2.1 A mapping $Q: \mathcal{H} \longrightarrow \mathcal{H}$ is said to be

(a) weakly hemicontinuous if $Q$ is upper semicontinuous from line segments in $\mathcal{H}$ to the weak topology of $\mathcal{H}$;

(b) sequentially weakly continuous if for each sequence $\left\{u_{n}\right\}$ in $\mathcal{H}$ with $\left\{u_{n}\right\} \rightarrow u\left(\left\{u_{n}\right\}\right.$ converges weakly to $u),\left\{Q\left(u_{n}\right)\right\}$ converges weakly to $Q(u)$.

Remark 2.2 It is easy to prove that if $Q: \mathcal{H} \longrightarrow \mathcal{H}$ is sequentially weakly continuous, then $Q$ must be weakly hemicontinuous.

It is well known that the following conclusion holds 
Lemma $2.3([6])$ A solution of $\operatorname{DVI}(\mathcal{C}, Q)$ is always a solution of $V I(\mathcal{C}, Q)$, provided that the operator $Q$ is, say, weakly hemicontinuous.

This is why we shall restrict our attention to $\operatorname{DVI}(\mathcal{C}, Q)$.

Remark 2.4 It is well-known that $u^{*} \in \mathcal{C}$ is a solution of (2.2) if and only if $u^{*}=P_{\mathcal{C}}\left(u^{*}-\right.$ $\left.\lambda Q\left(u^{*}\right)\right)$ for all $\lambda>0$.

Let $\mathcal{H}$ be a real Hilbert space. Given $x, y \in \mathcal{H}$, we define the closed line segment

$$
[x, y]=\{t x+(1-t) y: 0 \leq t \leq 1\} .
$$

The segments $(x, y],[x, y)$, and $(x, y)$ are defined analogously.

Definition 2.5 Let $\mathcal{C}$ be a nonempty and closed convex subset in $\mathcal{H}$, and let $Q: \mathcal{C} \longrightarrow \mathcal{H}$ be a mapping. The mapping $Q$ is said to be:

(a) strongly monotone on $\mathcal{C}$ with constant $\tau>0$ if for each pair of points $u, v \in \mathcal{C}$, we have

$$
\langle Q(u)-Q(v), u-v\rangle \geq \tau\|u-v\|^{2} ;
$$

(b) strictly monotone on $\mathcal{C}$ if for all distinct $u, v \in \mathcal{C}$, we have

$$
\langle Q(u)-Q(v), u-v\rangle>0
$$

(c) monotone on $\mathcal{C}$ if for each pair of points $u, v \in \mathcal{C}$, we have

$$
\langle Q(u)-Q(v), u-v\rangle \geq 0
$$

(d) pseudo-monotone on $\mathcal{C}$ if for each pair of points $u, v \in \mathcal{C}$, we have

$$
\langle Q(v), u-v\rangle \geq 0 \quad \Rightarrow \quad\langle Q(u), u-v\rangle \geq 0 ;
$$

(e) quasi-monotone on $\mathcal{C}$ if for each pair of points $u, v \in \mathcal{C}$, we have

$$
\langle Q(v), u-v\rangle>0 \quad \Rightarrow \quad\langle Q(u), u-v\rangle \geq 0 ;
$$

(f) (see [8]) semistrictly quasi-monotone on $\mathcal{C}$ if $Q$ is quasi-monotone on $\mathcal{C}$ and for all distinct of points $u, v \in \mathcal{C}$, we have that

$$
\langle Q(v), u-v\rangle>0 \quad \text { implies } \quad\langle Q(z), u-v\rangle>0, \quad \text { for some } z \in(0.5(u+v), u) \text {. }
$$

Remark 2.6 The following implications hold:

$$
(a) \Rightarrow(b) \Rightarrow(c) \Rightarrow(d) \Rightarrow(e) \text { and }(f) \Rightarrow(e) .
$$

But the reverse assertions are not true, in general. 


\section{Lemma 2.7}

(i) Each pseudo-monotone mapping $Q$ on $\mathcal{C}$ is semistrictly quasi-monotone on $\mathcal{C}$.

(ii) If $Q$ is quasi-monotone and affine on $\mathcal{H}$, i.e., $Q(u)=M u+q$, where $q \in \mathcal{H}$, and $M$ is a linear and bounded operator on $\mathcal{H}$, then $Q$ is semistrictly quasi-monotone on $\mathcal{H}$.

Proof (i) Suppose on the contrary that, for given $u, v \in \mathcal{C}$ with $u \neq v$, we have

$$
\langle Q(v), u-v\rangle>0 \Rightarrow\langle Q(z), u-v\rangle \leq 0, \quad \text { for all } z \in(0.5(u+v), u) .
$$

Since $z \in(0.5(u+v), u)$, it can be written as

$$
z=t\left(\frac{1}{2}(u+v)\right)+(1-t) u, \quad t \in(0,1) .
$$

This implies that $v-z=\left(1-\frac{1}{2} t\right)(v-u)$. Hence we have

$$
\langle Q(z), v-z\rangle \geq 0, \quad \text { for all } z \in(0.5(u+v), u) .
$$

By the pseudo-monotonicity of $Q$, it follows that

$$
\langle Q(v), v-z\rangle \geq 0, \quad \text { for all } z \in(0.5(u+v), u) .
$$

Hence we have

$$
\langle Q(v), u-v\rangle \leq 0
$$

which is a contradiction.

(ii) If $u, v \in \mathcal{H}$, with $u \neq v$, are such that $\langle M v+q, u-v\rangle>0$, then, by the quasimonotonicity of $Q$, we have

$$
\langle M u+q, u-v\rangle \geq 0
$$

Set $u_{\lambda}=\lambda v+(1-\lambda) u$. It follows that

$$
\left\langle M u_{\lambda}+q, u-v\right\rangle=\lambda\langle M v+q, u-v\rangle+(1-\lambda)(M u+q, u-v\rangle>0, \quad \forall \lambda \in(0,1) .
$$

Hence it holds for some $\lambda \in(0,0.5)$. For example, take $\lambda=0.3$, then $u_{0.3} \in(0.5(u+v), u)$ and $\left\langle M\left(u_{0.5}\right)+q, u-v\right\rangle>0$.

This completes the proof.

Proposition 2.8 ([14]) Let $\mathcal{C}$ be a nonempty, closed and convex subset of $\mathcal{H}$ and $Q$ : $\mathcal{C} \longrightarrow \mathcal{H}$ be a weakly hemicontinuous and semistrictly quasi-monotone mapping. Then $\operatorname{DVI}(\mathcal{C}, Q)(2.3)$ at least has one solution. 


\section{Weak convergence theorem}

In this section, we consider the problem $\operatorname{VI}(\mathcal{C}, Q)$ with $\mathcal{C}$ being a nonempty, closed, convex subset of $\mathcal{H}$, and $Q$ being semistrictly quasi-monotone on $\mathcal{H}$ and $\zeta$-Lipschitz continuous with $\zeta>0$ on $\mathcal{C}$.

Algorithm 3.1 Data: $u^{0} \in \mathcal{C}$ and $\left\{\lambda_{\ell}\right\} \in[a, b]$, where $0<a \leq b<\frac{1}{\zeta}$.

Step 0: Set $\ell=0$.

Step 1: If $u^{\ell}=P_{\mathcal{C}}\left(u^{\ell}-\lambda_{\ell} Q\left(u^{\ell}\right)\right)$ then stop.

Step 2: Otherwise, set

$$
\begin{aligned}
& \bar{u}^{\ell}=P_{\mathcal{C}}\left(u^{\ell}-\lambda_{\ell} Q\left(u^{\ell}\right)\right), \\
& u^{\ell+1}=P_{\mathcal{C}}\left(u^{\ell}-\lambda_{\ell} Q\left(\bar{u}^{\ell}\right)\right) .
\end{aligned}
$$

Replace $\ell$ by $\ell+1$; go to Step 1 .

Remark 3.2 Assume that $Q\left(u^{\ell}\right)=0$, then

$$
u^{\ell}=P_{\mathcal{C}}\left(u^{\ell}-\lambda_{\ell} Q\left(u^{\ell}\right)\right)
$$

and Algorithm 3.1 terminates at step $\ell$ with a solution $u^{\ell}$.

Therefore, without loss of generality, we can assume that $Q\left(u^{\ell}\right) \neq 0$ for all $\ell$, so that Algorithm 3.1 generates an infinite sequence.

Lemma $3.3([11,15])$ Assume that $Q$ is semistrictly quasi-monotone and $\zeta$-Lipschitz continuous on $\mathcal{C}$ and $\operatorname{Sol}(\mathcal{C}, Q)$ is nonempty. Let $u^{*}$ be a solution of $\operatorname{VI}(\mathcal{C}, Q)$. Then, for every $\ell \in N$, we have

$$
\left(1-\lambda_{\ell}^{2} \zeta^{2}\right)\left\|u^{\ell}-\bar{u}^{\ell}\right\|^{2} \leq\left\|u^{\ell}-u^{*}\right\|^{2}-\left\|u^{\ell+1}-u^{*}\right\|^{2}
$$

Theorem 3.4 Assume that $Q$ is semistrictly quasi-monotone on $\mathcal{H}$, sequentially weakly continuous and $\zeta$-Lipschitz continuous on $\mathcal{C}$. Then, the sequence $\left\{u^{\ell}\right\}$ generated by Algorithm 3.1 converges weakly to a solution of $V I(\mathcal{C}, Q)$.

Proof First we point out that, by the assumptions of Theorem 3.4, Remark 2.2, Lemma 2.3 and Proposition 2.8, we know that the solution $\operatorname{set} \operatorname{Sol}(\mathcal{C}, Q)$ is nonempty.

Since $0<a \leq \lambda_{\ell} \leq b<\frac{1}{\zeta}$, it holds that

$$
0<1-b^{2} \zeta^{2} \leq 1-\lambda_{\ell}^{2} \zeta^{2} \leq 1-a^{2} \zeta^{2}<1 .
$$

From Lemma 3.3, the sequence $\left\{u^{\ell}\right\}$ is bounded and

$$
\lim _{\ell \rightarrow \infty}\left\|u^{\ell}-\bar{u}^{\ell}\right\|=0
$$

Since $Q$ is Lipschitz continuous on $\mathcal{C}$, we have

$$
\left\|Q\left(u^{\ell}\right)-Q\left(\bar{u}^{\ell}\right)\right\| \leq \zeta\left\|u^{\ell}-\bar{u}^{\ell}\right\| .
$$


Hence

$$
\lim _{\ell \rightarrow \infty}\left\|Q\left(u^{\ell}\right)-Q\left(\bar{u}^{\ell}\right)\right\|=0
$$

Since $\left\{u^{\ell}\right\}$ is a bounded sequence in $\mathcal{H}$, there exists a subsequence $\left\{u^{\ell_{i}}\right\}$ of $\left\{u^{\ell}\right\}$ converging weakly to $\hat{u} \in \mathcal{C}$. Therefore

$$
\lim _{i \rightarrow \infty}\left\|u^{\ell_{i}}-\bar{u}^{\ell_{i}}\right\|=0
$$

also $\left\{\bar{u}^{\ell_{i}}\right\}$ converges weakly to $\hat{u}$.

Next we will show that $\hat{u} \in \operatorname{Sol}(\mathcal{C}, Q)$.

In fact, since

$$
\bar{u}^{\ell}=P_{\mathcal{C}}\left(u^{\ell}-\lambda_{\ell} Q\left(u^{\ell}\right)\right),
$$

by the projection characterization (2.1), it follows that

$$
\left\langle u^{\ell_{i}}-\lambda_{\ell_{i}} Q\left(u^{\ell_{i}}\right)-\bar{u}^{\ell_{i}}, v-\bar{u}^{\ell_{i}}\right\rangle \leq 0, \quad \forall v \in \mathcal{C},
$$

or equivalently,

$$
\frac{1}{\lambda_{\ell_{i}}}\left\langle u^{\ell_{i}}-\bar{u}^{\ell_{i}}, v-\bar{u}^{\ell_{i}}\right\rangle \leq\left\langle Q\left(u^{\ell_{i}}\right), v-\bar{u}^{\ell_{i}}\right\rangle \quad \forall v \in \mathcal{C} .
$$

This implies that

$$
\frac{1}{\lambda_{\ell_{i}}}\left\langle u^{\ell_{i}}-\bar{u}^{\ell_{i}}, v-\bar{u}^{\ell_{i}}\right\rangle+\left\langle Q\left(u^{\ell_{i}}\right), \bar{u}^{\ell_{i}}-u^{\ell_{i}}\right\rangle \leq\left\langle Q\left(u^{\ell_{i}}\right), v-u^{\ell_{i}}\right\rangle, \quad \forall v \in \mathcal{C} .
$$

Fixing $v \in \mathcal{C}$, letting $i \longrightarrow+\infty$ in the latter inequality, as well as remembering that

$$
\lim _{i \rightarrow \infty}\left\|u^{\ell_{i}}-\bar{u}^{\ell_{i}}\right\|=0
$$

and $\left.\lambda_{\ell} \in[a, b] \subset\right] 0, \frac{1}{\zeta}[$ for all $\ell$, we have

$$
\lim _{i \rightarrow \infty} \inf _{i \rightarrow \infty}\left\langle Q\left(u^{\ell_{i}}\right), v-u^{\ell_{i}}\right\rangle \geq 0 .
$$

Now we choose a sequence $\left\{\epsilon_{i}\right\}$ of positive numbers decreasing to 0 . For each $\epsilon_{i}$, we denote by $n_{i}$ the smallest positive integer such that

$$
\left\langle Q\left(u^{\ell_{j}}\right), v-u^{\ell_{j}}\right\rangle+\epsilon_{i}>0, \quad \forall j \geq n_{i},
$$

where the existence of $n_{i}$ follows from (3.3). Since $\left\{\epsilon_{i}\right\}$ is decreasing, it is easy to see that sequence $\left\{n_{i}\right\}$ is increasing. Furthermore, for each $i, Q\left(u^{\ell_{n_{i}}}\right) \neq 0$ and, setting

$$
v^{\ell_{n_{i}}}=\frac{Q\left(u^{\ell_{n_{i}}}\right)}{\left\|Q\left(u^{\ell_{n_{i}}}\right)\right\|^{2}},
$$


we have

$$
\left\langle Q\left(u^{\ell_{n_{i}}}\right), v^{\ell_{n_{i}}}\right\rangle=1 \quad \text { for each } i .
$$

It follows from (3.4) that for each $i$,

$$
\left\langle Q\left(u^{\ell_{n_{i}}}\right), v+\epsilon_{i} v^{\ell_{n_{i}}}-u^{\ell_{n_{i}}}\right\rangle>0 .
$$

Since $Q$ is semistrictly quasi-monotone, we have

$$
\left\langle Q\left(v+\epsilon_{i} v^{\ell_{n_{i}}}\right), v+\epsilon_{i} v^{\ell_{n_{i}}}-u^{\ell_{n_{i}}}\right\rangle>0 .
$$

On the other hand, since $\left\{u^{\ell_{i}}\right\}$ converges weakly to $\hat{u}$ as $i \longrightarrow \infty$, and $Q$ is sequentially weakly continuous on $\mathcal{C}$, it follows that $\left\{Q\left(u^{\ell_{i}}\right)\right\}$ converges weakly to $Q(\hat{u})$. We can suppose that $Q(\hat{u}) \neq 0$ (otherwise, $\hat{u}$ is a solution). Since the norm $\|\cdot\|$ is sequentially weakly lower semicontinuous, we have

$$
\|Q(\hat{u})\| \leq \liminf _{i \rightarrow \infty}\left\|Q\left(u^{\ell_{i}}\right)\right\| .
$$

Since $\left\{u^{\ell_{n_{i}}}\right\} \subset\left\{u^{\ell_{i}}\right\}$ and $\epsilon_{i} \longrightarrow 0$ as $i \longrightarrow 0$, we obtain

$$
0 \leq \lim _{i \rightarrow \infty}\left\|\epsilon_{i} v^{\ell_{n_{i}}}\right\|=\lim _{i \rightarrow \infty} \frac{\epsilon_{i}}{\left\|Q\left(u^{\ell_{n_{i}}}\right)\right\|} \leq \frac{0}{\|Q(\hat{u})\|}=0 .
$$

Taking the limit as $i \longrightarrow \infty$ in (3.5), we get

$$
\langle Q(v), v-\hat{u}\rangle \geq 0
$$

It follows from Lemma 2.3 and Remark 2.4 that $\hat{u} \in \operatorname{Sol}(\mathcal{C}, Q)$.

Finally, we show that sequence $\left\{u^{\ell}\right\}$ converges weakly to $\hat{u}$. To do this, it is sufficient to show that $\left\{u^{\ell}\right\}$ cannot have two distinct weak sequential limit points in $\operatorname{Sol}(\mathcal{C}, Q)$. Let $\left\{u^{\ell_{j}}\right\}$ be another subsequence of $\left\{u^{\ell}\right\}$ converging weakly to $\bar{u}$. We have to prove that $\hat{u}=\bar{u}$. As it has been proven above, $\bar{u} \in \operatorname{Sol}(\mathcal{C}, Q)$. From Lemma 3.3, the sequences $\left\{\left\|u^{\ell}-\hat{u}\right\|\right\}$ and $\left\{\left\|u^{\ell}-\bar{u}\right\|\right\}$ are monotonically decreasing and therefore converge. Since, for all $\ell \in N$,

$$
2\left\langle u^{\ell}, \bar{u}-\hat{u}\right\rangle=\left\|u^{\ell}-\hat{u}\right\|^{2}-\left\|u^{\ell}-\bar{u}\right\|^{2}+\|\bar{u}\|^{2}-\|\hat{u}\|^{2}
$$

we deduce that sequence $\left\{\left\langle u^{\ell}, \bar{u}-\hat{u}\right\rangle\right\}$ also converges. Setting

$$
\Im=\lim _{\ell \rightarrow \infty}\left\langle u^{\ell}, \bar{u}-\hat{u}\right\rangle
$$

and passing to the limit along $\left\{u^{\ell_{i}}\right\}$ and $\left\{u^{\ell_{j}}\right\}$ yields

$$
\Im=\langle\hat{u}, \bar{u}-\hat{u}\rangle=\langle\bar{u}, \bar{u}-\hat{u}\rangle .
$$

This implies that $\|\hat{u}-\bar{u}\|^{2}=0$, and therefore $\hat{u}=\bar{u}$. 


\section{A numerical example}

We consider a mapping $Q$ which is semistrictly quasi-monotone but not monotone.

Let $\mathcal{H}=l_{2}$ be the real Hilbert space whose elements are the square-summable sequences of real numbers, i.e., $\mathcal{H}=\left\{u=\left(u_{1}, u_{2}, \ldots, u_{i}, \ldots\right): \sum_{i=1}^{\infty}\left|u_{i}\right|^{2}<+\infty\right\}$. Let $\alpha, \beta \in \mathbb{R}$ be such that $\beta>\alpha>\frac{\beta}{2}>0$. Put

$$
\mathcal{C}_{\alpha}=\{u \in \mathcal{H}:\|u\| \leq \alpha\}, \quad Q_{\beta}(u)=(\beta-\|u\|) u,
$$

where $\alpha$ and $\beta$ are parameters. It is easy to see that $Q_{\beta}$ is sequentially weakly continuous on $\mathcal{H}$ and $\operatorname{Sol}\left(\mathcal{C}_{\alpha}, Q_{\beta}\right)=\{0\}$.

Next we prove that $Q_{\beta}: \mathcal{C}_{\alpha} \longrightarrow \mathcal{H}$ is Lipschitz continuous and semistrictly quasimonotone on $\mathcal{C}_{\alpha}$. In fact, for any $u, v \in \mathcal{C}_{\alpha}$, we have

$$
\begin{aligned}
\left\|Q_{\beta}(u)-Q_{\beta}(v)\right\| & =\|(\beta-\|u\|) u-(\beta-\|v\|) v\| \\
& =\|\beta(u-v)-\| u\|(u-v)-(\|u\|-\|v\|) v\| \\
& \leq \beta\|u-v\|+\|u\|\|u-v\|+|\|u\|-\|v\||\|v\| \\
& \leq \beta\|u-v\|+\alpha\|u-v\|+\|u-v\| \alpha \\
& =(\beta+2 \alpha)\|u-v\| .
\end{aligned}
$$

Hence, $Q_{\beta}$ is Lipschitz continuous on $\mathcal{C}_{\alpha}$ with the Lipschitz constant $\zeta=\beta+2 \alpha$. Let $u, v \in$ $\mathcal{C}_{\alpha}$ be such that

$$
\left\langle Q_{\beta}(u), v-u\right\rangle>0 .
$$

Then

$$
(\beta-\|u\|)\langle u, v-u\rangle>0 .
$$

Since $\|u\| \leq \alpha<\beta$, we have

$$
\langle u, v-u\rangle>0 .
$$

Hence,

$$
\begin{aligned}
\left\langle Q_{\beta}(v), v-u\right\rangle & =(\beta-\|v\|)\langle v, v-u\rangle \\
& \geq(\beta-\|v\|)(\langle v, v-u\rangle-\langle u, v-u\rangle) \\
& \geq(\beta-\alpha)\|u-v\|^{2}>0
\end{aligned}
$$

Thus, we shown that $Q_{\beta}$ is semistrictly quasi-monotone on $\mathcal{C}_{\alpha}$. It is worthy to stress that $Q_{\beta}$ is not monotone on $\mathcal{C}_{\alpha}$. To see this, it suffices to choose $u=\left(\frac{\beta}{2}, 0, \ldots, 0, \ldots\right)$, $v=(\alpha, 0, \ldots, 0, \ldots) \in \mathcal{C}_{\alpha}$ and note that

$$
\left\langle Q_{\beta}(u)-Q_{\beta}(v), u-v\right\rangle=\left(\frac{\beta}{2}-\alpha\right)^{3}<0 .
$$


Using the extragradient method for solving the semistrictly quasi-monotone variational inequality $\operatorname{VI}\left(\mathcal{C}_{\alpha}, Q_{\beta}\right)$, we choose $\lambda_{\ell}=\lambda \in\left(0, \frac{1}{\zeta}\right)=\left(0, \frac{1}{\beta+2 \alpha}\right)$ and any $u^{0} \in \mathcal{C}_{\alpha}$ as a starting point. The projection onto $\mathcal{C}_{\alpha}$ is explicitly calculated as

$$
P_{\mathcal{C}_{\alpha}} u= \begin{cases}u, & \text { if }\|u\| \leq \alpha, \\ \frac{\alpha u}{\|u\|}, & \text { otherwise. }\end{cases}
$$

Since, for all $\ell$,

$$
0<\lambda<\frac{1}{\beta+2 \alpha}<\frac{1}{\beta-\left\|u^{\ell}\right\|},
$$

we have

$$
\left\|u^{\ell}-\lambda Q_{\beta}\left(u^{\ell}\right)\right\|=\left(1-\lambda\left(\beta-\left\|u^{\ell}\right\|\right)\right)\left\|u^{\ell}\right\| \leq\left\|u^{\ell}\right\| \leq \alpha .
$$

Therefore,

$$
\bar{u}^{\ell}=P_{\mathcal{C}_{\alpha}}\left(u^{\ell}-\lambda_{\ell} Q\left(u^{\ell}\right)\right)=\left(1-\lambda\left(\beta-\left\|u^{\ell}\right\|\right)\right) u^{\ell} .
$$

Similarly, we can deduce that

$$
\left\|u^{\ell}-\lambda_{\ell} Q_{\beta}\left(\bar{u}^{\ell}\right)\right\| \leq \alpha .
$$

Indeed, we have

$$
u^{\ell}-\lambda_{\ell} Q_{\beta}\left(\bar{u}^{\ell}\right)=u^{\ell}-\lambda\left(\beta-\left\|\bar{u}^{\ell}\right\|\right)\left(1-\lambda\left(\beta-\left\|u^{\ell}\right\|\right)\right) u^{\ell} .
$$

Since

$$
\begin{aligned}
1 & -\lambda\left(\beta-\left\|\bar{u}^{\ell}\right\|\right)\left(1-\lambda\left(\beta-\left\|u^{\ell}\right\|\right)\right) \\
& =1-\lambda\left(\beta-\left\|\bar{u}^{\ell}\right\|\right)+\lambda^{2}\left(\beta-\left\|\bar{u}^{\ell}\right\|\right)\left(\beta-\left\|u^{\ell}\right\|\right) \\
& \geq 1-\lambda\left(\beta-\left\|\bar{u}^{\ell}\right\|\right)>0,
\end{aligned}
$$

we can write

$$
\left\|u^{\ell}-\lambda_{\ell} Q_{\beta}\left(\bar{u}^{\ell}\right)\right\|=\left[1-\lambda\left(\beta-\left\|\bar{u}^{\ell}\right\|\right)\left(1-\lambda\left(\beta-\left\|u^{\ell}\right\|\right)\right)\right]\left\|u^{\ell}\right\| \leq\left\|u^{\ell}\right\| \leq \alpha .
$$

This and (4.1) imply that

$$
\begin{aligned}
\left\|u^{\ell+1}\right\| & =\left\|P_{\mathcal{C}_{\alpha}}\left(u^{\ell}-\lambda_{\ell} Q_{\beta}\left(\bar{u}^{\ell}\right)\right)\right\| \\
& =\left\|u^{\ell}-\lambda\left(\beta-\left\|\bar{u}^{\ell}\right\|\right) \bar{u}^{\ell}\right\| \\
& =\left[1-\lambda\left(\beta-\left\|\bar{u}^{\ell}\right\|\right)\left(1-\lambda\left(\beta-\left\|u^{\ell}\right\|\right)\right)\right]\left\|u^{\ell}\right\| .
\end{aligned}
$$


We have

$$
\begin{aligned}
\lambda\left(\beta-\left\|\bar{u}^{\ell}\right\|\right)\left(1-\lambda\left(\beta-\left\|u^{\ell}\right\|\right)\right) & =\lambda\left(\beta-\left\|\bar{u}^{\ell}\right\|\right)\left(1-\lambda \beta+\lambda\left\|u^{\ell}\right\|\right) \\
& \geq \lambda\left(\beta-\left\|\bar{u}^{\ell}\right\|\right)(1-\lambda \beta) \\
& =\lambda\left(\beta-(1-\lambda \beta)\left\|u^{\ell}\right\|-\lambda\left\|u^{\ell}\right\|^{2}\right)(1-\lambda \beta) .
\end{aligned}
$$

Considering the function $q(x)=\beta-(1-\lambda \beta) x-\lambda x^{2}$ with $x \in[0, \alpha]$, it is easy to see that $q$ is decreasing on $[0, \alpha]$. Therefore, the minimal value of $q$ is

$$
\beta-(1-\lambda \beta) \alpha-\lambda \alpha^{2},
$$

which is attained at $x=\alpha$. Combining this with (4.3) and (4.2) yields

$$
\begin{aligned}
\left\|u^{\ell+1}\right\| & \leq\left(1-\lambda\left(\beta-(1-\lambda \beta) \alpha-\lambda \alpha^{2}\right)(1-\lambda \beta)\right)\left\|u^{\ell}\right\| \\
& \leq\left(1-\left(\lambda \beta-\lambda \alpha+\lambda^{2} \beta \alpha-\lambda^{2} \alpha^{2}\right)(1-\lambda \beta)\right)\left\|u^{\ell}\right\| \\
& =[1-(\beta-\alpha) \lambda(1+\alpha \lambda)(1-\lambda \beta)]\left\|u^{\ell}\right\| .
\end{aligned}
$$

We claim that

$$
\varrho=(\beta-\alpha) \lambda(1+\alpha \lambda)(1-\lambda \beta) \in(0,1)
$$

Indeed, since $\alpha<\beta$ and $0<\lambda<\frac{1}{\beta+2 \alpha}$, we have $\varrho>0$. To verify that $\varrho<1$, it is sufficient to show that $(\beta-\alpha) \lambda(1+\alpha \lambda)<1$. Since $\frac{\beta}{2}<\alpha<\beta$ and $0<\lambda<\frac{1}{\beta+2 \alpha}$, we have

$$
\begin{aligned}
(\beta-\alpha) \lambda(1+\alpha \lambda) & <(\beta-\alpha) \frac{1}{\beta+2 \alpha}\left(1+\frac{\alpha}{\beta+2 \alpha}\right) \\
& <\frac{\beta}{2(\beta+\beta)}\left(1+\frac{\beta}{\beta+\beta}\right)=\frac{3}{8} .
\end{aligned}
$$

This implies that $\varrho \in(0,1)$, and we can deduce from (4.4) that

$$
\left\|u^{\ell}\right\| \leq(1-\varrho)^{\ell}\left\|u^{0}\right\|, \quad \text { for all } \ell \in \mathbb{N}
$$

This means that the sequence $\left\{u^{\ell}\right\}$ converges strongly to 0 , the unique solution of $V I\left(\mathcal{C}_{\alpha}, Q_{\beta}\right)$.

\section{Acknowledgements}

We would like to express our thanks to the anonymous referees and the editor for their constructive comments and suggestions, which greatly improved this article.

\section{Funding}

This work was supported by the National Natural Science Foundation of China (Grant 11361070) and supported by the Scientific Research Fund of Science and Technology Department of Sichuan Province (No. 2018-JY0340). This work was also supported by the Natural Science Foundation of Center for General Education, China Medical University, Taichung, Taiwan. 


\section{Competing interests}

The authors declare that there is no conflict of interest regarding the publication of this paper.

\section{Authors' contributions}

All authors contributed equally and significantly in writing this article. All authors read and approved the final manuscript.

\section{Author details}

${ }^{1}$ Center for General Education, China Medical University, Taichung, Taiwan. ²Department of Mathematics, Jazan

University, Jazan, Kingdom of Saudi Arabia. ${ }^{3}$ Yunnan University of Finance and Economics, Kunming, China.

${ }^{4}$ Department of Mathematics, Yibin University, Yibin, China.

\section{Publisher's Note}

Springer Nature remains neutral with regard to jurisdictional claims in published maps and institutional affiliations.

Received: 15 February 2019 Accepted: 18 March 2019 Published online: 22 March 2019

\section{References}

1. Agarwal, R.P., Ahmad, M.K., Salahuddin: Existence of solutions to weakly generalized vector F-implicit variational inequalities. Discontinuity, Nonlinearity, Complexity 1(3), 225-235 (2012)

2. Agarwal, R.P., Ahmad, M.K., Salahuddin: Hybrid type generalized multivalued vector complementarity problems. Ukr. Math. J. 65(1), 6-20 (2013)

3. Censor, Y., Gibali, A., Reich, S.: The subgradient extragradient method for solving variational inequalities in Hilbert space. J. Optim. Theory Appl. 148, 318-335 (2011)

4. Censor, Y., Gibali, A., Reich, S.: Strong convergence of subgradient extragradient methods for the variational inequality problem in Hilbert space. Optim. Methods Softw. 26, 827-845 (2011)

5. Censor, Y., Gibali, A., Reich, S.: Extensions of Korpelevich extragradient method for the variational inequality problem in Euclidean space. Optimization 61,1119-1132 (2012)

6. Daniilidis, A., Hadjisavvas, N.: Characterization of nonsmooth semistrictly quasiconvex and strictly quasiconvex functions. J. Optim. Theory Appl. 102, 525-536 (1999)

7. Facchinei, F., Pang, J.-S.: Finite-Dimensional Variational Inequalities and Complementarity Problems, Vols. I and II. Springer, New York (2003)

8. Hadjisavvas, N., Chaible, S.: On strong pseudomonotonicity and (semi)strict quasimonotonicity. J. Optim. Theory Appl. 79(1), 139-155 (1993)

9. Karamardian, S., Schaible, S.: Seven kinds of monotone maps. J. Optim. Theory Appl. 66, 37-46 (1990)

10. Khanh, P.D.: A new extragradient method for strongly pseudomonotone variational inequalities. Numer. Funct. Anal. Optim. 37, 1131-1143(2016)

11. Khanh, P.D.: A modified extragradient method for infinite-dimensional variational inequalities. Acta Math. Vietnam. 41, 251-263 (2016)

12. Kim, J.K., Salahuddin, Lim, W.H.: An iterative algorithm for generalized mixed equilibrium problems and fixed points of nonexpansive semigroups. J. Appl. Math. Phys. 5, 276-293 (2017)

13. Kinderlehrer, D., Stampacchia, G.: An Introduction to Variational Inequalities and Their Applications. Academic Press, New York (1980)

14. Konnov, I.V.: On quasimonotone variational inequalities I. J. Optim. Theory Appl. 99(1), 165-181 (1998)

15. Korpelevich, G.M.: The extragradient method for finding saddle points and other problems. Metody 12, 747-756 (1976)

16. Lee, B.S., Salahuddin: Scalarization techniques for set valued vector variational type inequalities. Arch. J. Math. 2(2), 121-129 (2012)

17. Tam, N.N., Yao, J.-C., Yen, N.D.: Solution methods for pseudomonotone variational inequalities. J. Optim. Theory Appl. $138,253-273$ (2008)

18. Vuong, P.T.: On the weak convergence of the extragradient method for solving pseudo-monotone variational inequalities. J. Optim. Theory Appl. 176, 399-409 (2018)

19. Zhu, D.L., Marcotte, P.: Co-coercivity and its role in the convergence of iterative schemes for solving variational inequalities. SIAM J. Control Optim. 6, 714-726 (1996)

\section{Submit your manuscript to a SpringerOpen ${ }^{\circ}$ journal and benefit from:}

- Convenient online submission

- Rigorous peer review

- Open access: articles freely available online

- High visibility within the field

- Retaining the copyright to your article

Submit your next manuscript at $\boldsymbol{\text { springeropen.com }}$ 